
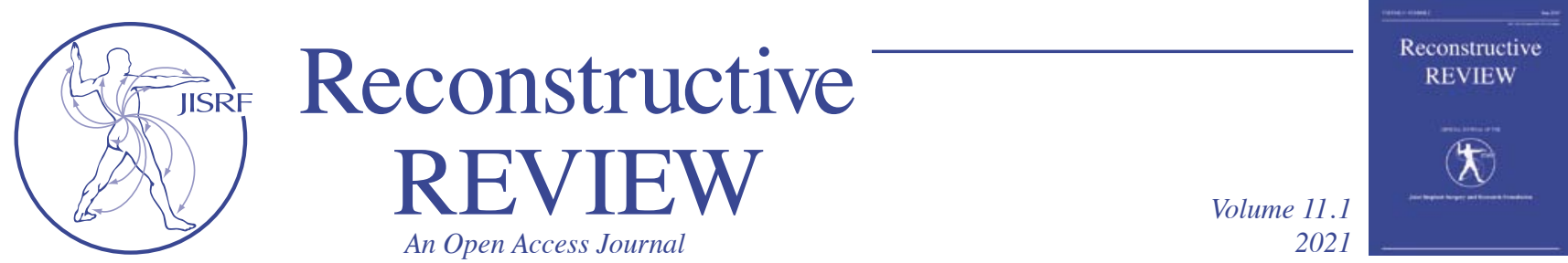

\title{
Intravenous versus Intra-Articular Tranexamic Acid in Primary Total Hip Arthroplasty: A Prospective Randomised Double Blinded Non-Inferiority Trial
}

Hasan, $A^{1}$; Campbell, $D^{1,2}$; Lewis, $P^{1,2}$

\section{Abstact}

Background: Tranexamic acid (TXA) has been shown to be effective in reducing post-operative blood loss after hip replacement surgery. Clinicians can be reluctant to administer intravenous (IV) TXA to high risk patients and intra-articular (IA) administration has been proposed as an alternative mode of delivery. This study was conducted to compare the efficacy of IV versus IA administration of TXA.

Methods: This prospective, double blinded, randomised non-inferiority trial, compared 69 patients undergoing primary total hip arthroplasty (THA) who received either 3 doses of $15 \mathrm{mg} / \mathrm{kg}$ of IV TXA or $3 \mathrm{~g}$ of IA TXA after capsular closure. The primary outcomes were change in $\mathrm{Hb}$ and the rate of blood transfusion. The secondary outcome was the rate of VTE.

Results: The mean haemoglobin level change from pre-operative to day 1 post-operative for the IV group was $26.7 \mathrm{~g} / \mathrm{L}$ and for IA group was $27.3 \mathrm{~g} / \mathrm{L}$. No statistically significant difference was detected between the two groups $(\mathrm{p}=0.82)$. No patients required a transfusion or developed a VTE.

Conclusions: IA administration of TXA can be equally effective as IV in the reduction of blood loss and the prevention of post-operative anaemia in primary THA.

\section{Background}

Tranexamic acid (TXA), an antifibrinolytic agent that has been in clinical use since the 1960s, has become commonly used in orthopaedic surgery over the last decade, often as a component of a multimodal blood loss management strategy. Post operative anaemia is common following hip arthroplasty surgery with some studies reporting transfusion rates of $24.4 \%$ prior to the introduction of TXA [1]. Allogeneic transfusions are associated with complications including immunosuppression, perioperative infections and transfusion-related reactions $[\underline{2}, \underline{3}]$. Among patients with acute coronary syndromes, there is an increased risk of myocardial infarction and death $[\underline{4}, \underline{5}]$. Furthermore, anaemia may slow a patient's postoperative rehabilitation and prolong the length of hospital stay with additional costs [] $]$.

Intravenous (IV) administration of TXA during arthroplasty surgery has been shown to decrease peri-operative blood loss, thereby reducing transfusion rates [-10]. The decreased transfusion requirements and length of stay is cost effective and the routine use of TXA is recommended $[\underline{11}, \underline{12}]$.

Keywords: Tranexamic Acid, Hip Arthroplasty, Intravenous, Intra-Articular, Topical, Blood Loss Level of Evidence: II 
The proposed advantages of a single dose IA (intra-articular) TXA administration include targeted therapeutic delivery with lower plasma concentration of TXA [13] potentially mitigating the risk of systemic adverse events and eliminating the requirement for repeated post-operative IV administration. Common medical conditions such as previous venous thromboembolism, renal impairment, cerebrovascular and myocardial disease preclude universal administration of IV TXA despite no evidence of risk to these patients [14]. This population with medical co-morbidities would benefit from the prevention of post-operative anaemia.

IA administration of TXA has been compared to IV use in total hip arthroplasty with variable results $[\underline{12}, \underline{15}, \underline{16}]$. North et al and Xie et al observed that IA was not as effective as IV administration at reducing post-operative haemoglobin drop $[\underline{12}, \underline{16}]$. However, all 3 studies observed equal transfusion rates amongst the IV and IA cohorts [15]. All three studies used only a single dose of IV TXA. It is standard at our institution to give 3 doses at 8 hourly intervals, a multi-dosing approach which has been associated with improved effectiveness [7]. Given the uncertainty in the literature, this study aims to confirm if IA can be an effective and equal substitute for IV multi-dose administration.

The purpose of this prospective, randomised non-inferiority trial was to compare the efficacy of intravenous (IV) versus intra-articular (IA) administration of TXA in patients undergoing primary total hip arthroplasty by comparing the post-operative haemoglobin $(\mathrm{Hb})$ change and transfusion requirement.

\section{Materials \& Methods}

\section{Patients}

The study design was a prospective double blinded, randomised non-inferiority trial. Between June 2016 and October 2017, patients were recruited from Wakefield Orthopaedic Clinic, a metropolitan private hospital. All patients undergoing primary total hip arthroplasty for osteoarthritis were included. Independent Ethics Board approval was granted from the Calvary Health Care Adelaide Human Research Ethics Committee (Ref no. 16-CHREC-F001) and the trial was registered with the Australian New Zealand Clinical Trials Registry (ACTRN 12616000907448).

Exclusion criteria included those with acquired defective colour vision, previous subarachnoid haemorrhage, active intravascular clotting, or hypersensitivity to TXA (which are the product specified contra-indications), as well as those with a hypercoaguable tendency (history of arterial or venous thromboembolic disease such as CVA, DVT or PE, or fibrinolytic disorder requiring anti-fibrinolytic treatment), coagulopathy (PLT $<150$, INR $>1.4$ ) or cognitive impairment. Patients on continued anticoagulation agents were excluded to eliminate this confounding factor. Patients with renal impairment (creatinine $>120 \mathrm{mmol} / \mathrm{L}$ ) were also excluded to keep the administered dose the same for all patients. The exclusion criteria was based on previous similar studies investigating the effectiveness of TXA in order to produce a comparable cohort.

A consistent blood management protocol was used. The protocol included pre-operative $\mathrm{Hb}$ assessment and optimization if required. All patients were advised to cease anti-inflammatory medications and health supplements ten days prior to surgery. Spinal anaesthetic was used with normotensive or hypotensive control. Normothermia was maintained. TXA was administered to all patients at $15 \mathrm{mg} /$ $\mathrm{kg}$ approximated to $1 \mathrm{~g}$ aliquots which translated to a total dose of $3 \mathrm{~g}$ for all patients. Post operative $\mathrm{Hb}$ was measured on the morning the day after surgery. We adhered to an evidence-based transfusion protocol, whereby patients were transfused if they had a $\mathrm{Hb}<70 \mathrm{~g} / \mathrm{L}$, or $\mathrm{Hb}<100 \mathrm{~g} / \mathrm{L}$ with symptoms of cardiovascular or cerebrovascular ischemia $[\underline{17}, \underline{18}]$.

All surgeries were performed by 2 experienced arthroplasty surgeons using their usual surgical technique and implant. Drains were not used. VTE prophylaxis included bilateral pneumatic calf compression devices and $100 \mathrm{mg}$ Aspirin commencing day 1 post-operative for a minimum of four weeks. Median length of stay was 4 days for both surgeons. Both surgeons had similar surgical techniques and post-operative protocols.

\section{Randomisation and Administration}

Allocation was determined using an electronic randomizer in blocks of 20 and envelopes were then numbered consecutively. Envelopes were opened by the anaesthetist in the operating theatre who prepared the treatment and placebo IV and IA solutions. Patients were assigned to one of two groups; the IV group received IV TXA 15mg/ $\mathrm{kg} 8$ hourly for three doses commencing at induction of anaeasthesia plus a placebo of $40 \mathrm{ml}$ normal saline injected intra-articular in a single sterile syringe. The IA group received $3 \mathrm{~g}$ of TXA mixed with $40 \mathrm{ml}$ normal saline in a single sterile syringe that was administered IA plus a placebo of similar volume IV normal saline. IA application was performed by injecting the $40 \mathrm{~mL}$ solution intra-articular after capsular closure. The second and third doses of IV solution (TXA or saline) was prescribed by the anaesthetist and administered by the ward nurses. The surgeon and patient remained blinded throughout this process. 


\section{Outcomes}

The primary outcome was the $\mathrm{Hb}$ change comparing preoperative $\mathrm{Hb}$ and day 1 postoperative $\mathrm{Hb}$. Secondary outcomes were the rate of transfusion and the incidence of inpatient venous thromboembolism. A restrictive investigation protocol was routine practice and patients were investigated with limb venous ultrasonography if they demonstrated clinical symptoms and signs of VTE.

\section{Statistical Analysis}

Sample size was calculated using a twosample means test, setting a non-inferiority margin for $\mathrm{Hb}$ change of $8 \mathrm{~g} / \mathrm{L}$ with a standard deviation of $12.6 \mathrm{~g} / \mathrm{L}$. These parameters have been used by others $[\underline{19}, \underline{20}]$. In order to achieve $90 \%$ power at the 0.05 level of significance, 32 patients per study arm were required.

Unadjusted and adjusted linear regression models were performed to detect a statistically significantly different $(\mathrm{P}$ value $<0.05$ ) change in $\mathrm{Hb}$. Fisher's Exact Test was used to find an association between TXA treatment group and transfusion requirement.

\section{Results}

\section{Patient Cohort}

Of the 86 patients deemed eligible, 13 patients were excluded and the remaining 73 patients were randomised to either IV or IA application (Figure 1). The final analysis consisted of 35 patients in the IV and 34 patients in the IA treatment arm.

The patient demographics and surgical parameters are listed in Table 1. The two cohorts were similar. The average age was 67.6 years. The majority of hip arthroplasties were uncemented, performed via a posterior approach.

\section{Haemoglobin change}

There was a mean $\mathrm{Hb}$ drop of $26.7 \mathrm{~g} / \mathrm{L}$ for the IV group and $27.3 \mathrm{~g} / \mathrm{L}$ for the IA group (Table 2). An unadjusted linear regression of $\mathrm{Hb}$ change versus TXA treatment group, adjusting for $\mathrm{Hb}$ pre-surgery, did not show a statistically significant difference in $\mathrm{Hb}$ change between the 2 cohorts $(\mathrm{P}$ value $=0.7665)$. An adjusted linear regression of $\mathrm{Hb}$ change versus TXA treatment group, adjusting for $\mathrm{Hb}$ pre-surgery, age, BMI, surgeon, approach, stem fixation and operation time was performed. Adjusting for these variables, there was no statistically significant difference
Figure 1. Participant flow

Declined to participate $(n=3)$ Missed recruitment $(n=10)$

Randomized $(n=73)$
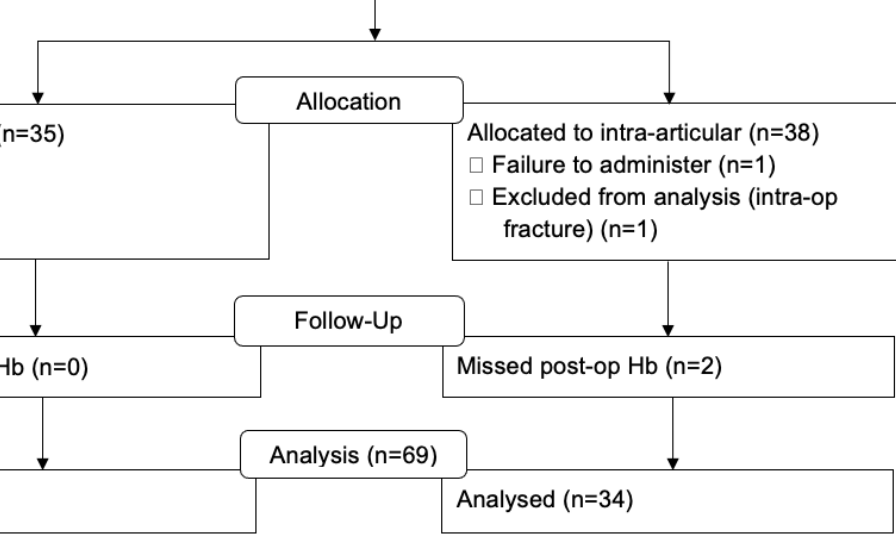

Table 1: Baseline Characteristics of Study Population

\begin{tabular}{|c|c|c|c|}
\hline & IV $(n=35)$ & IA $(n=34)$ & Total $(n=69)$ \\
\hline \multicolumn{4}{|c|}{ Patient Demographics } \\
\hline Male & 14 & 14 & $28(41 \%)$ \\
\hline Female & 21 & 20 & $41(59 \%)$ \\
\hline Average age & $70.0 \pm 10.2$ & $65.2 \pm 10.5$ & $67.6 \pm 10.5$ \\
\hline BMI & $27.7 \pm$ & $29.1 \pm 6.1$ & $28.4 \pm 5.5$ \\
\hline \multicolumn{4}{|l|}{ ASA } \\
\hline 1 & 2 & 3 & $5(7 \%)$ \\
\hline 2 & 20 & 16 & $36(52 \%)$ \\
\hline 3 & 13 & 14 & $27(41 \%)$ \\
\hline 4 & 0 & 1 & $1(1 \%)$ \\
\hline \multicolumn{4}{|c|}{ Surgical Parameters } \\
\hline Surgeon 1 & 25 & 26 & $51(74 \%)$ \\
\hline Surgeon 2 & 10 & 8 & $18(26 \%)$ \\
\hline Posterior & 33 & 30 & $63(91 \%)$ \\
\hline Anterior & 2 & 4 & $6(9 \%)$ \\
\hline Uncemented & 30 & 30 & $60(87 \%)$ \\
\hline Cemented & 5 & 4 & $9(13 \%)$ \\
\hline \multicolumn{4}{|c|}{ Average op time } \\
\hline (mins) & $72.6 \pm 18.4$ & $74.5 \pm 15.1$ & $73.5 \pm 16.8$ \\
\hline
\end{tabular}

Table 2: Outcomes of the Two Groups - Mean and Standard deviations

\begin{tabular}{|l|c|c|c|}
\hline & IV $(\mathbf{n = 3 5})$ & IA $(\mathbf{n = 3 4})$ & P value \\
\hline Pre-op $\mathrm{Hb}(\mathrm{g} / \mathrm{L})$ & $138.1 \pm 10.5$ & $137.6 \pm 12.0$ & 0.86 \\
\hline Post-op $\mathrm{Hb}(\mathrm{g} / \mathrm{L})$ & $111.5 \pm 13.3$ & $110.3 \pm 14.7$ & 0.73 \\
\hline Hb drop $(\mathrm{g} / \mathrm{L})$ & $26.7 \pm 12.2$ & $27.3 \pm 11.8$ & 0.82 \\
\hline Transfused & 0 & 0 & 0.00 \\
\hline DVT & 0 & 0 & 0.00 \\
\hline
\end{tabular}


in $\mathrm{Hb}$ change between the two treatment groups ( $\mathrm{P}$ value $=0.6298$ ).

\section{Transfusion and Venous thromboembolism rate}

None of the patients in this study required a transfusion. Additionally, none of the patients developed an inpatient venous thromboembolism.

\section{Discussion}

We found that IA TXA was equally effective as IV administration in total hip arthroplasty in restricting post-operative blood loss as measured by drop in $\mathrm{Hb}$. In this study a single $3 \mathrm{~g}$ dose of IA TXA administered during surgery was not inferior to the three separate $15 \mathrm{mg} / \mathrm{kg}$ doses of IV TXA. There was no clinically significant difference in the post-operative haemoglobin decrease, post-operative haemoglobin value or transfusion requirements.

Strategies to limit blood loss associated with arthroplasty surgery are increasingly used by arthroplasty surgeons and TXA is commonly used for coagulation cascade manipulation [1]. Multiple studies have been published with universal demonstration of its clinical efficacy, however the ideal route and timing of administration are less clear. IA administration is a potential option to mitigate systemic TXA effects. Wong et al found the plasma concentration of TXA after topical application was 70\% less than the same dose of TXA administered by IV injection [13]. While there is good evidence to support the use of TXA in all patients, with no evidence of adverse effects, there is some reluctance for routine use of IV TXA amongst clinicians less familiar with the data due to theoretical concerns of a prothrombotic effect.

No clinical studies, meta-analyses or registry studies have demonstrated an association with an increased risk of VTE or arterial thrombosis [21] but many clinical trials have excluded patients considered to be of increased risk. Whilst there is no data to suggest an increased risk with universal TXA use [14], the converse is less clear: i.e. whether there is an increased risk of medical complications by withholding TXA in the 'high risk' patient group. Two recent clinical studies addressing the use of TXA in the 'high risk' group have not demonstrated an increased complication rate [22,23]. IV TXA was not associated with an increased risk of VTE in patients with a history of VTE [22]. Further, a Danish registry study has demonstrated not only a reduced risk of arterial thrombosis (myocardial infarction and ischaemic stroke), but reduced rate of allcause mortality, if TXA is still administered in these patients [24].
Despite the paucity of evidence of harm, Patel et al [25] observed that TXA was considered a contraindication in over $25 \%$ of patients. Further, Ho et al [26] observed $6 \%$ of patients had a failure of a universal administration protocol citing concern from clinical staff (such as anaesthesia) and were denied TXA treatment.

There are limited prospective studies assessing the efficacy of intra-articular TXA in hip arthroplasty patients and comparison of these is difficult due to the variety of doses and dosing regimens used. Konig et al and Wei and Wei used three separate $1 \mathrm{~g}$ injections instilled after acetabular preparation, after femoral broaching and before/ after fascial closure $[\underline{15}, \underline{27}]$. Wei and Wei reported $3 \mathrm{~g}$ IA was equally effective as the same dose IV at reducing total blood loss (958 $\mathrm{ml}$ [IV] vs $963 \mathrm{ml}$ [IA]) and the transfusion rate $(5.94 \%$ [IV], $5.88 \%$ [IA]), but did not report on the effect on $\mathrm{Hb}$ change [15]. North et al used a lower dose of $2 \mathrm{~g}$ TXA, and bathed the wound with TXA after component placement and observed an inferior blood sparing effect when compared to the IV group (Hb drop $3.1 \mathrm{~g} / \mathrm{dL}$ [IV] vs $3.5 \mathrm{~g} / \mathrm{dL}$ [IA], transfusion rate $11 \%$ [IV] vs $18 \%$ [IA] [12]. $\mathrm{Xie}$ et al observed a significantly smaller $\mathrm{Hb}$ change in a $1.5 \mathrm{~g}$ IV dose group compared to $3 \mathrm{~g}$ IA group $(2.98 \mathrm{~g} / \mathrm{dL}$ [IV] vs 3.36g/dL [IA]) [16]. They also showed a combination of $1 \mathrm{~g}$ IV and $2 \mathrm{~g}$ IA administration was more effective than either IA or IV in isolation (Hb drop $3.89 \mathrm{~g} / \mathrm{dL}[\mathrm{IV}$ and IA]) [16] . Similarly, Yi et al found that a combination of IV and IA administration was more effective that IV delivery alone at reducing postoperative bleeding and the transfusion rate (16\% [IV] vs $2 \%$ [IV and IA] [료].

This study found an overall smaller $\mathrm{Hb}$ change and transfusion rate across both treatment groups compared to previous studies which may be the result of the effectiveness of our strict blood management protocol. Unlike North et al and Xie et al, we found IA application was not inferior to IV in preventing post-operative anaemia.

This study design differs from others being a non-inferiority trial comparing a single $3 \mathrm{~g}$ TXA IA injection following capsule closure (a contemporary dosage regime) to three $15 \mathrm{mg} / \mathrm{kg}$ IV doses (the manufacturer recommended regime). This is also consistent with the method used in a study of knee arthroplasty patients (3g TXA IA compared to three divided IV doses) which also demonstrated no difference in blood loss $[\underline{29}, \underline{30}]$.

We acknowledge the limitations of this study. This study was powered to detect a primary outcome of the day 1 postoperative fall of $\mathrm{Hb}$ level setting a non-inferiority margin of $8 \mathrm{~g} / \mathrm{L}$ that was validated with a post hoc analysis. The requirement for blood transfusion could not be studied due to the low frequency of this event (as anticipated with the inclusion of TXA and a contemporary blood manage- 
ment protocol) [17]. Similarly we did not design the study to detect medical or surgical complications and we did not compare clinical outcomes. Our results on VTE rate are consistent with previously published data; it is a relatively uncommon event and difficult to demonstrate a statistically significant association with any route of TXA administration.

\section{Conclusion}

This study shows IA administration is an effective alternative to IV administration for TXA. IA administration may have a particular place for patients where there are concerns about possible systemic side-effects from IV use.

\section{Acknowledgements}

We thank Christine Schultz for assisting with data collection and logistics and Suzanne Edwards, Data Management and Analysis Centre at the University of Adelaide, for her help with the statistical analysis of the collected data.

\section{References}

1. Newman C, Tran P, McGregor S, Bramley D. Patient blood management strategies in total hip and knee arthroplasty. Current Orthopaedic Practice. 2018;29(1):316. doi: 10.1097/bco.0000000000000577. PubMed PMID: 01337441-20180100000008.

2. Hill GE, Frawley WH, Griffith KE, Forestner JE, Minei JP. Allogeneic blood transfusion increases the risk of postoperative bacterial infection: a meta-analysis. Journal of Trauma and Acute Care Surgery. 2003;54(5):908-14.

3. Lemaire R. Strategies for blood management in orthopaedic and trauma surgery. Journal of Bone \& Joint Surgery, British Volume. 2008;90(9):1128-36.

4. Yang X, Alexander KP, Chen AY, Roe MT, Brindis RG, Rao SV, et al. The implications of blood transfusions for patients with non-ST-segment elevation acute coronary syndromes: results from the CRUSADE National Quality Improvement Initiative. Journal of the American College of Cardiology. 2005;46(8):1490-5.

5. Rao SV, Eikelboom JA, Granger CB, Harrington RA, Califf RM, Bassand J-P. Bleeding and blood transfusion issues in patients with non-ST-segment elevation acute coronary syndromes. European heart journal. 2007;28(10):1193-204.

6. Myers E, Grady P, Dolan A. The influence of preclinical anaemia on outcome following total hip replacement. Archives of orthopaedic and trauma surgery. 2004;124(10):699-701.

7. Sukeik M, Alshryda S, Haddad F, Mason J. Systematic review and meta-analysis of the use of tranexamic acid in total hip replacement. Journal of Bone \& Joint Surgery, British Volume. 2011;93(1):39-46.

8. Alshryda S, Sarda P, Sukeik M, Nargol A, Blenkinsopp J, Mason J. Tranexamic acid in total knee replacement A systematic review and meta-analysis. Journal of Bone \& Joint Surgery, British Volume. 2011;93(12):1577-85.

9. Yang Z-G, Chen W-P, Wu L-D. Effectiveness and safety of tranexamic acid in reducing blood loss in total knee arthroplasty: a meta-analysis. The Journal of Bone \& Joint Surgery. 2012;94(13):1153-9.

10. Zhou X-d, Tao L-j, Li J, Wu L-d. Do we really need tranexamic acid in total hip arthroplasty? A meta-analysis of nineteen randomized controlled trials. Archives of orthopaedic and trauma surgery. 2013;133(7):1017-27.

11. Tuttle JR, Ritterman SA, Cassidy DB, Anazonwu WA, Froehlich JA, Rubin LE. Cost benefit analysis of topical tranexamic acid in primary total hip and knee arthroplasty. The Journal of arthroplasty. 2014;29(8):1512-5.

12. North WT, Mehran N, Davis JJ, Silverton CD, Weir RM, Laker MW. Topical vs intravenous tranexamic acid in primary total hip arthroplasty: a double-blind, randomized controlled trial. The Journal of arthroplasty. 2016;31(5):1022-6.
13. Wong J, Abrishami A, El Beheiry H, Mahomed NN, Davey JR, Gandhi R, et al. Topical application of tranexamic acid reduces postoperative blood loss in total knee arthroplasty: A randomized, controlled trial. Journal of Bone and Joint Surgery - Series A. 2010;92(15):2503-13. PubMed PMID: 2010651223.

14. Fillingham YA, Ramkumar DB, Jevsevar DS, Yates AJ, Bini SA, Clarke HD, et al. Tranexamic Acid Use in Total Joint Arthroplasty: The Clinical Practice Guidelines Endorsed by the American Association of Hip and Knee Surgeons, American Society of Regional Anesthesia and Pain Medicine, American Academy of Orthopaedic Surgeons, Hip Society, and Knee Society. The Journal of arthroplasty. 2018;33(10):3065.

15. Wei W, Wei B. Comparison of topical and intravenous tranexamic acid on blood loss and transfusion rates in total hip arthroplasty. Journal of Arthroplasty. 2014;29(11):2113-6. PubMed PMID: 25155138.

16. Xie J, Ma J, Yue C, Kang P, Pei F. Combined use of intravenous and topical tranexamic acid following cementless total hip arthroplasty: a randomised clinical trial. Hip International. 2016;26(1):36-42.

17. Bruce W, Campbell D, Daly D, Isbister J. Practical recommendations for patient blood management and the reduction of perioperative transfusion in joint replacement surgery. ANZ journal of surgery. 2013;83(4):222-9.

18. Carson JL, Terrin ML, Noveck H, Sanders DW, Chaitman BR, Rhoads GG, et al. Liberal or restrictive transfusion in high-risk patients after hip surgery. New England Journal of Medicine. 2011;365(26):2453-62.

19. Alshryda S, Mason J, Sarda P, Nargol A, Cooke N, Ahmad H, et al. Topical (intra-articular) tranexamic acid reduces blood loss and transfusion rates following total hip replacement: a randomized controlled trial (TRANX-H). Journal of Bone \& Joint Surgery - American Volume. 2013;95(21):1969-74. PubMed PMID: 24196467.

20. Fillingham YA, Kayupov E, Plummer DR, Moric M, Gerlinger TL, Della Valle CJ. The James A. Rand Young Investigator's Award: a randomized controlled trial of oral and intravenous tranexamic acid in total knee arthroplasty: the same efficacy at lower cost? The Journal of arthroplasty. 2016;31(9):26-30.

21. Ker K, Edwards P, Perel P, Shakur H, Roberts I. Effect of tranexamic acid on surgical bleeding: systematic review and cumulative meta-analysis. Bmj. 2012;344:e3054.

22. Sabbag OD, Abdel MP, Amundson AW, Larson DR, Pagnano MW. Tranexamic acid was safe in arthroplasty patients with a history of venous thromboembolism: a matched outcome study. The Journal of arthroplasty. 2017;32(9):S246-S50.

23. Whiting DR, Gillette BP, Duncan C, Smith H, Pagnano MW, Sierra RJ. Preliminary results suggest tranexamic acid is safe and effective in arthroplasty patients with severe comorbidities. Clinical Orthopaedics and Related Research ${ }^{\circledR}$. 2014;472(1):66-72.

24. Dastrup A, Pottegård A, Hallas J, Overgaard S. Perioperative tranexamic acid treatment and risk of cardiovascular events or death after total hip arthroplasty: a population-based cohort study from national Danish databases. JBJS 2018;100(20):1742-9.

25. Patel JN, Spanyer JM, Smith LS, Huang J, Yakkanti MR, Malkani AL. Comparison of intravenous versus topical tranexamic acid in total knee arthroplasty: a prospective randomized study. Journal of Arthroplasty. 2014;29(8):1528-31. PubMed PMID: 24768543.

26. Ho A. Is it time we routinely give tranexamic acid in total joint arthroplasty? Annual Scientific Meeting; 10th October 2019; Canberra: Australian Orthopaedic Association.

27. Konig G, Hamlin BR, Waters JH. Topical tranexamic acid reduces blood loss and transfusion rates in total hip and total knee arthroplasty. The Journal of arthroplasty. 2013;28(9):1473-6.

28. Yi Z, Bin S, Jing Y, Zongke Z, Pengde K, Fuxing P. Tranexamic acid administration in primary total hip arthroplasty: a randomized controlled trial of intravenous combined with topical versus single-dose intravenous administration. JBJS. 2016;98(12):983-91.

29. Goyal N, Chen DB, Harris IA, Rowden NJ, Kirsh G, MacDessi SJ. Intravenous vs intra-articular tranexamic acid in total knee arthroplasty: a randomized, doubleblind trial. The Journal of arthroplasty. 2017;32(1):28-32.

30. Soni A, Saini R, Gulati A, Paul R, Bhatty S, Rajoli SR. Comparison between intravenous and intra-articular regimens of tranexamic acid in reducing blood loss during total knee arthroplasty. Journal of Arthroplasty. 2014;29(8):1525-7. PubMed PMID: 24814890. 


\section{SUBMISSION HISTORY}

Submitted: July 22, 2020

Reviewed: August 8, 2020

Revised November 10, 2020

Accepted: Dcember 4, 2020

Published: January 30, 2021

\section{AUTHOR AFFILIATIONS}

Afsana P Hasan, MBBS'; David Campbell, MBBS, PhD, FRACS, FAOrthA',2;

Peter Lewis, MBBS, FRACS, FAOrthA ${ }^{1,2}$

1 Centre of Orthopaedics and Trauma Research, University of Adelaide, Adelaide, South Australia, Australia

2 Wakefield Orthopaedic Clinic, Adelaide, South Australia, Australia (Direct inquires to Afsana Hasan, afsana.hasan@gmail.com)

\section{AUTHOR DISCLOSURES}

- The authors certify that no benefits or funds were received in direct or indirect support of this article.

COPYRIGHT \& OPEN ACCESS

(C) 2021 Hasan, Campbell, Lewis. All rights reserved. Authors retain copyright and grant the journal right of first publication with the work. Reconstructive Review is an open access publication

and follows the Creative Commons Attribution-NonCommercial

CC BY-NC. This license allows anyone to download works,

OPEN ACCESS build upon the material, and share them with others for non-

commercial purposes as long as they credit the senior author, Reconstructive Review, and the Joint Implant Surgery \& Research Foundation (JISRF). An example credit would be: "Courtesy of (senior author's name), Reconstructive Review, JISRF, Chagrin Falls, Ohio". 